\title{
Impaired B cell immunity in acute myeloid leukemia patients after chemotherapy
}

\author{
Meghali Goswami ${ }^{1 *+}$ (D), Gabrielle Prince ${ }^{2 \dagger}$, Angelique Biancotto ${ }^{3}$, Susan Moir ${ }^{4}$, Lela Kardava ${ }^{4}$, \\ Brian H. Santich ${ }^{4}$, Foo Cheung ${ }^{3}$, Yuri Kotliarov ${ }^{3}$, Jinguo Chen $^{3}$, Rongye Shi ${ }^{3}$, Huizhi Zhou ${ }^{3}$, Hana Golding ${ }^{5}$, \\ Jody Manischewitz ${ }^{5}$, Lisa King ${ }^{5}$, Lauren M. Kunz ${ }^{6}$, Kimberly Noonan², Ivan M. Borrello², B. Douglas Smith² \\ and Christopher S. Hourigan ${ }^{1}$
}

\begin{abstract}
Background: Changes in adaptive immune cells after chemotherapy in adult acute myeloid leukemia (AML) may have implications for the success of immunotherapy. This study was designed to determine the functional capacity of the immune system in adult patients with AML who have completed chemotherapy and are potential candidates for immunotherapy.

Methods: We used the response to seasonal influenza vaccination as a surrogate for the robustness of the immune system in $10 \mathrm{AML}$ patients in a complete remission post-chemotherapy and performed genetic, phenotypic, and functional characterization of adaptive immune cell subsets.

Results: Only 2 patients generated protective titers in response to vaccination, and a majority of patients had abnormal frequencies of transitional and memory B-cells. B-cell receptor sequencing showed a B-cell repertoire with little evidence of somatic hypermutation in most patients. Conversely, frequencies of T-cell populations were similar to those seen in healthy controls, and cytotoxic T-cells demonstrated antigen-specific activity after vaccination. Effector T-cells had increased PD-1 expression in AML patients least removed from chemotherapy.

Conclusion: Our results suggest that while some aspects of cellular immunity recover quickly, humoral immunity is incompletely reconstituted in the year following intensive cytotoxic chemotherapy for AML. The observed B-cell abnormalities may explain the poor response to vaccination often seen in AML patients after chemotherapy. Furthermore, the uncoupled recovery of B-cell and T-cell immunity and increased PD-1 expression shortly after chemotherapy might have implications for the success of several modalities of immunotherapy.
\end{abstract}

Keywords: Adaptive immunity, Leukemia, B-cells, T-cells, Influenza vaccination, Immunotherapy

\section{Background}

Acute myeloid leukemias (AML) encompass a heterogeneous group of oligoclonal hematological malignancies characterized by rapid, uncontrolled proliferation of leukemic blasts in the bone marrow and peripheral blood [1]. Initial treatment for AML typically includes intensive cytotoxic chemotherapy intended to achieve

\footnotetext{
*Correspondence: meg.goswami@nih.gov

${ }^{+}$Meghali Goswami and Gabrielle Prince are first authors

${ }^{1}$ Myeloid Malignancies Section, Hematology Branch, National Heart,

Lung and Blood Institute, National Institutes of Health, 10 Center Drive

Room 10CRC 5-5216, Bethesda, MD 20814-1476, USA

Full list of author information is available at the end of the article
}

a morphological complete remission (CR) in the bone marrow, but current standard of care regimens have poor clinical outcomes, as over half of AML patients will relapse and only a quarter will survive past 5 years [2].

Allogeneic hematopoietic stem cell transplantation (allo-HSCT) is a consolidative immunotherapy strategy to prevent relapse but is itself associated with significant morbidity and mortality [3]. Nevertheless, given the poor clinical outcomes with chemotherapy alone, the addition of other immunotherapeutic modalities to prevent relapse after completion of cytotoxic chemotherapy is an appealing prospect [4-6]. Cancer vaccination against tumor-specific antigens is a logical immunotherapeutic 
strategy to prevent relapse by further priming an immune response, and induction of leukemia-associated antigenspecific CD8+ T-cells have been observed in response to peptide vaccination, though responses are often short-lived [7-10]. Vaccination to retain remission in AML patients has produced conflicting results, with some groups reporting long-term efficacy and sustained immune response, while others report deletion of highavidity CD8+ T-cell clones and unsustainable responses [11-14].

Though immunotherapeutic strategies to maintain remission after patients receive intensive chemotherapy are logical and being tested in clinical trials [6], there is an incomplete description of the state of the adaptive immune system in AML patients who have completed chemotherapy. Previous work on peripheral blood lymphocyte recovery in AML after induction demonstrated a skewing of the T-cell compartment towards peripherally expanded oligoclonal activated T-regulatory cells (T-regs) in the time immediately following chemotherapy [15]. Yet, the functional capacity of the immune system in AML patients after the completion of intensive chemotherapy is largely unknown, and this has important implications for the success of any subsequent immunotherapy intended to prevent relapse, especially cancer vaccination and immune checkpoint blockade meant to augment endogenous cellular-mediated antileukemic responses.

The intent of the present study was to quantify phenotypic and functional immune abnormalities in a cohort of AML patients that would be considered ideal candidates for immunotherapies intended to prevent relapse. To eliminate the potential confounding effects of testing novel leukemia vaccines of unknown antigenic potency, we instead studied the well-characterized seasonal influenza vaccine with well-known efficacy profiles in healthy subjects. Recent studies have illuminated the presence of influenza vaccine-induced cell signatures that are predictive of robust immune responses across a spectrum of healthy individuals $[16,17]$. Influenza is a significant cause of morbidity and mortality in patients with hematological malignancies $[18,19]$. Because perturbations in the adaptive immune system after chemotherapy have severe implications for the success of any immunotherapy, especially vaccine-based modalities, we sought to interrogate the recovery and presence or absence of immune cell populations potentially predictive of response to vaccination in AML patients. We performed comprehensive phenotypic, genetic, and functional immune profiling immediately before and 30 days after the seasonal influenza vaccination in ten AML patients in a first remission after consolidation chemotherapy.

\section{Methods}

\section{Study design}

This study was approved by Johns Hopkins University Institutional Review Board (IRB) and conducted in accordance with the Declaration of Helsinki. All patients signed written consent. Patients were eligible for this study if they had completed treatment for AML at least 3 and no more than 156 weeks prior to enrollment, were judged to be in a complete remission, had not yet received the 2012-2013 seasonal influenza vaccine but were due to receive it as standard of care, and had no prior adverse reactions to vaccinations. Patients received the 2012-2013 trivalent inactivated seasonal influenza vaccine. Peripheral blood was collected on the day of vaccination (prior to vaccination) and 30 days post-vaccination. Ten age- and sex-matched healthy donors (HD) were recruited on an IRB approved healthy donor protocol and provided peripheral blood samples.

\section{Sample collection and processing}

Peripheral blood mononuclear cells (PBMCs) were purified by Ficoll-Hypaque density centrifugation from whole blood delivered into Leucosep tubes (Greiner Bio-One, Radnor, PA, USA). Cells were viably cryopreserved in a controlled rate freezer and distributed for RNA isolation, flow cytometry, and ELISPOT assays. Serum from whole blood was also separated using SST tubes, cryopreserved, and aliquoted for microneutralization assays. Whole blood was also drawn into PAXgene Blood RNA tubes (PreAnalytiX, Feldbachstrasse, Switzerland) as an additional source of nucleic acids. Detailed standard operating procedures are included in Additional file 1.

\section{Microneutralization assay}

Viral-neutralizing activity in AML patients who received the influenza vaccination was analyzed via microneutralization assay with MDCK cells based on the methods developed by the pandemic influenza reference laboratories of the Center for Disease Control and Prevention [20]. Neutralizing antibody titers were measured against seasonal influenza type A H3N2, H1N1, and type B vaccine strains. Human sera from patients at baseline and day 30 were tested at a starting dilution of $1: 20$, followed by serial twofold dilutions. Those that were negative for antibody titers were assigned a titer of $<20$. All sera were tested in triplicate, and the geometric mean was used in analysis. A fourfold or greater rise in neutralizing antibody titer at day 30 over baseline was considered a positive response (seroconversion).

\section{Flow cytometry}

High-dimensional flow cytometry for comprehensive leukocyte immunophenotyping was performed. Cell suspensions (from viably frozen cell suspensions) were stained 
as previously described [21, 22], and the staining panel is detailed in Additional file 2: Table S1. Acquisition was performed using a Becton-Dickinson LSRFortessa (BD, San Jose, CA, USA) equipped with five lasers $(355,407,488$, 532, and $633 \mathrm{~nm}$ wavelengths) with 22 PMT detectors, optimized as described by Perfetto et al. [23]. Data were acquired using DIVA 8 software (BD) and we recorded a minimum of 50,000 CD4+ T-cells to be able to accurately assess minor cell populations. Post-acquisition analysis was performed using Flowjo version 9.6.2 (Treestar Inc., San Carlos, CA, USA). For analysis, debris and doublets were excluded using light scatter measurements; subsequently, viability stain was used to ensure that only viable cell subsets were included. All major cell populations were identified based on the co-expression of CD45 and a pan lineage marker as described [17, 22]. Each cell population was represented as percentages of the parent population.

Cell-surface PD-1, TIM3, CTLA4, and 4-1BB expression on CD8+ T-cell subsets was also assessed on the AML patients at baseline and HD. Detailed methods can be found in Additional file 1.

\section{T-cell and B-cell ELISPOT}

IFNY T-cell ELISPOT assays were used to assess CD8+ T-cell function by quantifying the number of cytokinesecreting T-cells (CSCs), and IgG and IgA B-cell ELISPOT assays were used to assess B-cell functionality by enumerating antibody-secreting B-cells (ASCs). Extended methods can be found in Additional file 1.

\section{Gene expression analysis using microarray}

RNA was amplified from 300 ng of total RNA, and singlestrand sense cDNA was reverse transcribed, biotinylated, and hybridized to the GeneChip Human Gene 1.0 ST Arrays (Affymetrix, Santa Clara, CA, USA) after fragmentation. The arrays were washed and stained on a GeneChip Fluidics Station 450; scanning was carried out with the GeneChip Scanner 3000 and image analysis was done with the Affymetrix GeneChip Command Console (AGCC) software. Resulting CEL files were processed with Affymetrix Power Tools for probe set summarization, normalization, and log2-transformation (RMA with sketch quantile normalization). Ingenuity Pathway Analysis software (Ingenuity Systems, Redwood City, CA, USA) and Toppgene Suite were used for functional interpretation of gene expression data.

\section{Deep sequencing of the B-cell receptor heavy chain}

Amplification and sequencing of the B-cell receptor (BCR) immunoglobulin heavy (IGH) chain complementaritydetermining region 3 (CDR3) region was performed using the immunoSEQ platform (Adaptive Biotechnologies ${ }^{\odot}$, Seattle, WA, USA). The somatically rearranged CDR3 region was amplified from $1.5 \mu \mathrm{g}$ genomic DNA isolated from PAXgene tubes using a two-step, amplification biascontrolled multiplex PCR approach as described elsewhere $[24,25]$, including the Additional file 1.

\section{Statistical analyses}

Two-tailed Mann-Whitney U tests were used to compare variables in 2 sample groups, and F-tests were used to compare data distribution between 2 sample groups. KruskalWallis analysis of variance (ANOVA) tests were used to test more than 2 sample groups simultaneously. All analyses were performed using Prism software (GraphPad, La Jolla, CA, USA), Microsoft Excel, and R/Conductor. Standard deviation of the mean (SEM) values were calculated to summarize frequencies of immune subsets in HD. For all comparisons, $\mathrm{p}<0.05$ was considered statistically significant, and multiple testing corrections were performed where appropriate.

\section{Results}

\section{Patient demographics}

Demographics and clinical information at baseline of the ten AML patients are summarized in Table 1. The average age of enrolled patients was 53 years (range 28-69) with a median age of 57 years. All patients were in a first complete remission (CR1) following consolidation chemotherapy, and the average time elapsed from the last chemotherapy treatment was 37 weeks (range 4-148 weeks). Complete blood count details are detailed in Additional file 2: Table S2.

\section{Poor responses of AML patients after chemotherapy to influenza vaccination}

Only 2 of 10 of AML patients seroconverted (fourfold or higher antibody titer at day 30 compared to baseline) after vaccination to one or more of the influenza strains (AML responders, or AML-R) as assessed by microneutralization assay (Fig. 1a). One responder (AML 06) was 148 weeks post-chemotherapy, and the other (AML 10) had acute promyelocytic leukemia (APL). Some non-responders (AML-NR) had pre-existing titers but demonstrated no rise in neutralizing antibody titer after vaccination. These results were further confirmed using B-cell ELISPOT with the influenza vaccine formulation for 2012-2013. Patients 06 and 10 were the only two patients with influenza-specific IgA and IgG antibody secreting cells (ASCs) after influenza vaccination (Fig. 1b), and neither showed high levels of non-specific ASCs (Additional file 3: Figure S1).

\section{Global immunome assessment reveals differences between healthy subjects and AML patients after chemotherapy}

To look for global differences between AML patients after chemotherapy (before influenza vaccination) and 
Table 1 Patient clinical characteristics

\begin{tabular}{|c|c|c|c|c|c|c|c|c|}
\hline Patient & $\begin{array}{l}\text { Age } \\
\text { at baseline } \\
\text { (years)/sex }\end{array}$ & Diagnosis & Cytogenetics & Induction & Consolidation & $\begin{array}{l}\text { Remission } \\
\text { status }\end{array}$ & $\begin{array}{l}\text { Weeks since end } \\
\text { of chemother- } \\
\text { apy }\end{array}$ & $\begin{array}{l}\text { Baseline } \\
\text { ALC (/cu } \\
\mathrm{mm})\end{array}$ \\
\hline AML 01 & $67 / F$ & M5 & trisomy 8 , inv(16) & AcIVP16 & $\mathrm{HiDAc} \times 1$ & CR1 & 49 & 1660 \\
\hline AML 02 & $69 / F$ & MDS to AML & $46 X X$ & $7+3$ & Moderate dose Ac & CR1 & 10 & 750 \\
\hline AML 03 & $52 / \mathrm{M}$ & N/A & $46 \mathrm{XY}, \mathrm{NPM} 1+$ & FLAM & FLAM & CR1 & 45 & 2530 \\
\hline AML 04 & $48 / F$ & N/A & $\operatorname{inv}(16)$ & AcDVP16 & $\mathrm{HiDAc} \times 4$ & CR1 & 19 & 810 \\
\hline AML 05 & $28 / F$ & N/A & $46 X X$ & AcDVP16 & AcDAc & CR1 & 26 & 1860 \\
\hline AML 06 & $58 / \mathrm{M}$ & $\mathrm{N} / \mathrm{A}$ & $\mathrm{t}(8 ; 21)$ & AcDVP16 & $\mathrm{HiDAc} \times 1, \mathrm{AcDAc}$ & CR1 & 148 & 2710 \\
\hline AML 07 & $55 / \mathrm{M}$ & M5 & 46 XY, FLT3-D835+, NPM1+ & AcDVP16 & $\mathrm{HiDAc} \times 4$ & CR1 & 31 & 1160 \\
\hline AML 08 & $66 / M$ & M5 & $46 \mathrm{XY}, \mathrm{NPM1} 1+$ & AcDVP16 & $\mathrm{HiDAc} \times 4$ & CR1 & 8 & 650 \\
\hline AML 09 & $63 / \mathrm{M}$ & M4 & $46 \mathrm{XY}, \mathrm{NPM1+}$ & FLAM & FLAM & CR1 & 34 & 730 \\
\hline AML 10 & $28 / \mathrm{M}$ & M3 (APL) & $\mathrm{t}(15 ; 17)$, FLT3-ITD+ & ATRA/D & AcDArsenic & CR1 & 4 & 1440 \\
\hline
\end{tabular}

AML subtype under diagnosis defined by the French-American-British (FAB) classification of AML. MDS myelodysplastic syndrome, APL acute promyelocytic leukemia, ITD internal tandem duplication, NPM1 nucleophosmin, FLT3 fms-like tyrosine kinase, ITD internal tandem duplication, CR1 first complete remission, ALC absolute lymphocyte count. A/Ac cytarabine, I idarubicin, VP16 etoposide, FL flavoperidol, M mitoxantrone, D daunorubicin, ATRA all trans retinoic acid, HiD high dose

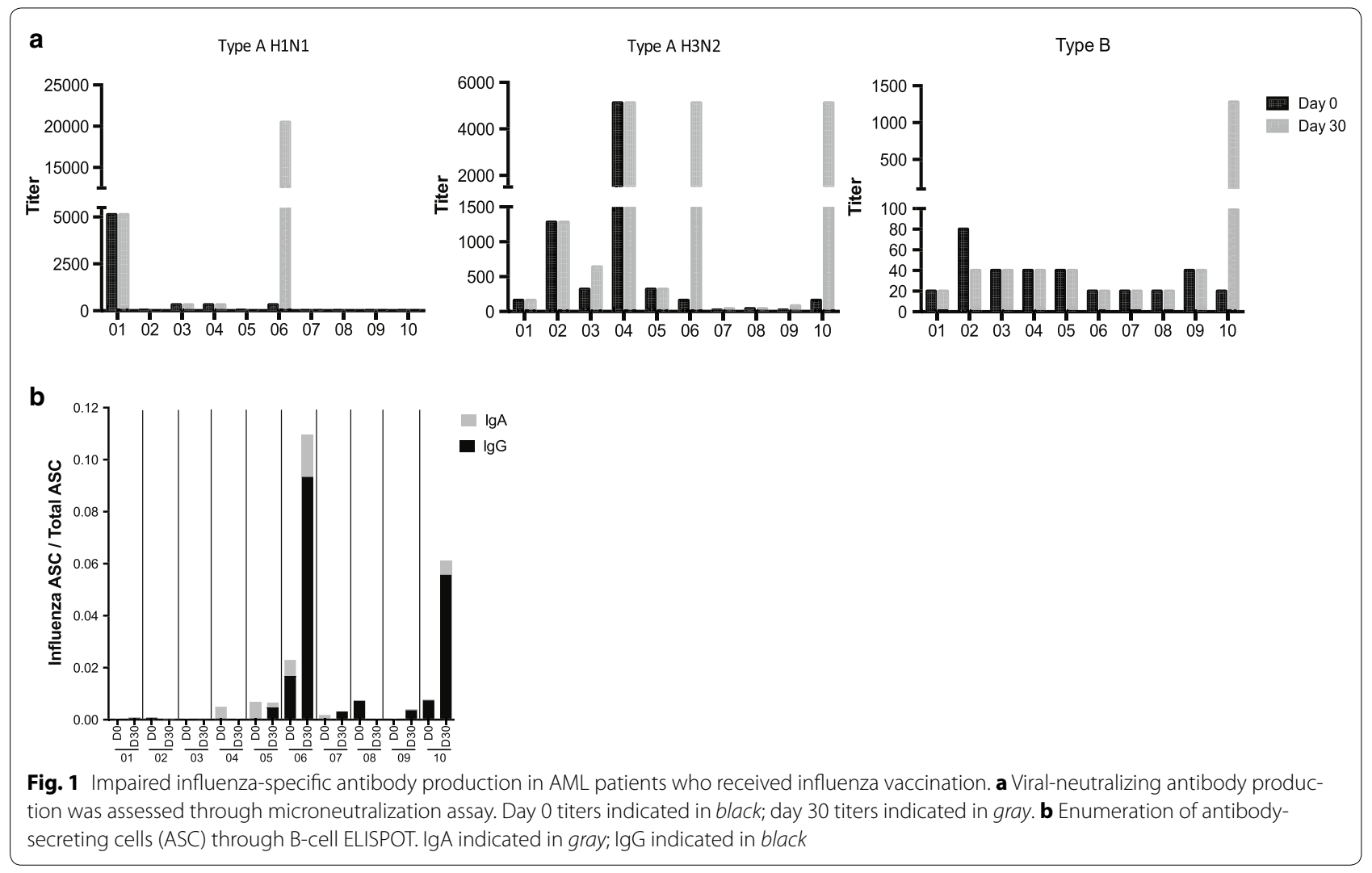

age- and gender-matched HDs, deep immunophenotyping of immune cell populations was conducted. In total, 93 cell populations were analyzed, listed in Additional file 2: Table S3. To better visualize global differences in cell population frequencies, we plotted the frequencies of the cell populations from the AML patients normalized to the average frequency seen in HD, which illustrates relatively over-expressed and under-expressed immune subsets (Fig. 2a). We identified 7/38 (18.4\%) T-cell populations, $3 / 11(27.3 \%)$ monocyte and dendritic cell populations, and 11/44 (25.0\%) B-cell populations where the mean frequencies in AML $(\mathrm{n}=10)$ and HD $(\mathrm{n}=10)$ significantly differed from one another, highlighted in Fig. 2a. We also specifically focused on eight AML patients who did not 
respond to influenza vaccination (AML-NR) in comparison to HD and found 4/38 (10.5\%) T-cell populations where the mean frequencies significantly differed, 3 of which overlapped with T-cell populations identified when considering all 10 AML patients. Two of 3 monocyte and dendritic cell populations also had significantly different mean frequencies in AML-NR vs HD. By these same metrics, we identified 10/44 (22.7\%) B-cell populations where mean frequencies significantly differed between AMLNR and HD, 9 of which overlapped with B-cell populations identified from looking at all 10 AML patients. We also found 4/11 (10.5\%), 4/11 (36.4\%), and 7/44 (15.9\%) T-cell, monocyte and dendritic cell, and B-cell populations, respectively, were the variances in cell frequencies between AML and HD significantly differed from one another. All data are summarized in Additional file 2: Table S4. Together, these data highlight heterogeneity across our AML cohort, a greater perturbation in the B-cell compartment in AML patients after chemotherapy, and cell populations that may be especially affected in AML patients who do not respond to influenza vaccination.

We next considered differences in global gene expression profiles to investigate any underlying genetic pathways potentially driving the responses we observed by analyzing microarray data derived from PBMCs of AML patients and HD. Supervised clustering between the eight AML-NR at baseline versus HD identified 1871 genes that were significantly differentially expressed, with 846 genes up-regulated and 1025 genes down-regulated in AML-NR (Fig. 2b). Gene set enrichment analysis (GSEA) of positively differentially expressed genes (DEG) in AML-NR revealed 111 gene sets with significant $p$-values (adjusted for multiple testing), including many immunerelated, signaling, and cell death pathways (Additional file 2: Table S5, Additional file 3: Figure S2). Specifically looking at leukocyte development and activation-related gene sets revealed several genes that drove their positive regulation, including LGALS9 (Additional file 2: Table S6). We noted that the two AML responders had markedly different gene expression profiles from one another and from HD. GSEA analysis of the 240 genes that were upregulated in AML 06 versus HD revealed an upregulation of many of the same gene sets up-regulated in the
AML-NR (Additional file 2: Table S7). The upregulation of genes related to the immune response, immune development and activation, cell signaling, and cell death in AML-NR indicate that despite irregularities in cell frequencies, the immune system in these patients is highly active and likely undergoing immune reconstitution, even in the patient (AML 06) who was in remission for 3 years at the time of enrollment to this study.

\section{Increased transitional B-cell and lower memory B-cell frequencies in AML patients after chemotherapy compared with healthy donors}

We examined the frequencies of differing cell populations in greater detail, specifically considering both mean frequencies and variances in frequency distribution between AML and HD, to better characterize the data between groups. Frequencies of total CD19+ B-cells (ID80) were comparable between AML and HD (mean frequency \pm SEM: $13.3 \% \pm 7.3$ vs. $14.5 \% \pm 2.3, \mathrm{p}=0.8$ ), and they ranged from 3.2 to $25.7 \%$ in AML and 5.9 to $28.4 \%$ in HD (Fig. 3a, left panel). Similarly, frequencies of naïve B-cells (ID106) in AML mirrored those seen in HD $(58.4 \% \pm 6.6$ vs. $60.4 \% \pm 4.8)$ with similar ranges (Fig. 3a, middle panel). However, there were significantly higher frequencies of transitional B-cells (ID90) in AML patients than in $\mathrm{HD}(35.5 \% \pm 6.8$ vs. $15.5 \% \pm 6.7$, $\mathrm{p}<0.05)$ (Fig. 3a, right panel). The range of these cells was narrow in HD (9.0-20.7\%) and strikingly wide in AML patients (15.2-77.6\%), such that the variance significantly differed between HD and AML $(\mathrm{p}<0.05)$. We saw a reduction of total memory B-cells (ID94) in AML at baseline compared to $\mathrm{HD}(5.8 \% \pm 1.7$ vs. $24.1 \% \pm 5.2$, $\mathrm{p}<0.01$ ) (Fig. 3b, left panel). This reduction was also mirrored in the mature B-cell IgA+ (ID83) $(4.8 \% \pm 0.7$ vs. $11.2 \% \pm 1.6, \mathrm{p}<0.01)$ and IgG+ (ID84) $(3.2 \% \pm 0.8$ vs. $5.9 \% \pm 1.0, \mathrm{p}<0.05)$ compartments (Fig. 3b, middle and right panels).

Figure 3c shows relative proportions of immature/ transitional, naïve, and memory B-cells in all AML compared to HD, illustrating the abnormally high frequencies of immature/transitional and markedly low frequencies of memory B-cells. Consideration of some constituent subpopulations of the memory B-cell compartment

\footnotetext{
(See figure on next page.)

Fig. 2 Global immunome analysis reveals differences between AML patients after chemotherapy and age/sex matched healthy donors. a Box and whisker plots of multi-parameter flow cytometry data. Frequencies of subpopulations T-cells, B-cells, dendritic cells, and monocytes were tabulated as a percentage of the average frequency of each cell population in HD. Blue dotted line indicates the normalized average in HD. Red plots mark populations where mean cell frequencies significantly $(p<0.05$ with multiple testing correction) differed between AML $(n=10)$ and HD $(n=10)$. b Heat map generated from a supervised clustering of gene expression data. Each column represents an individual subject; each row represents a gene. First 8 columns are AML-NR, next 2 columns are AML-R, and last 10 columns are HD. All data represents baseline gene expression. The genes were filtered using criteria of absolute value of log-fold-change higher than 0.2 and FDR-adjusted $p$ value less than 0.05 . Up-and down-regulated genes are noted by colors indicated in color key
} 
a

(10000-1000-10

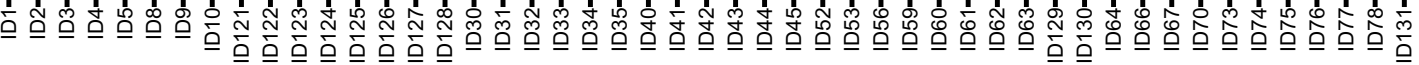

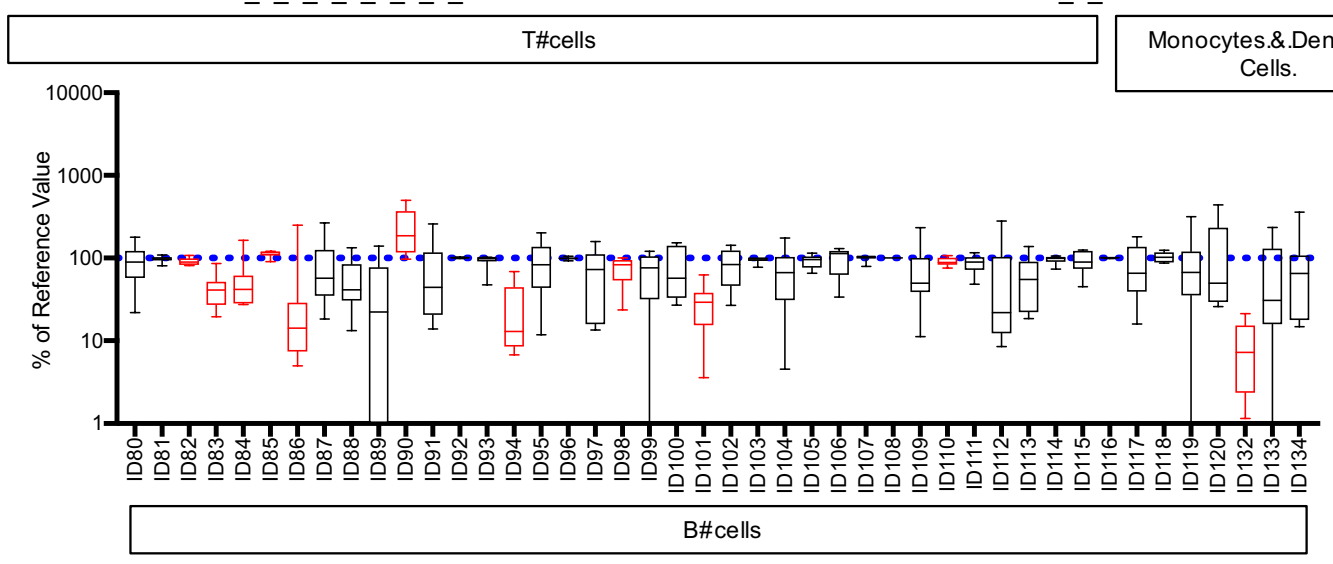

b
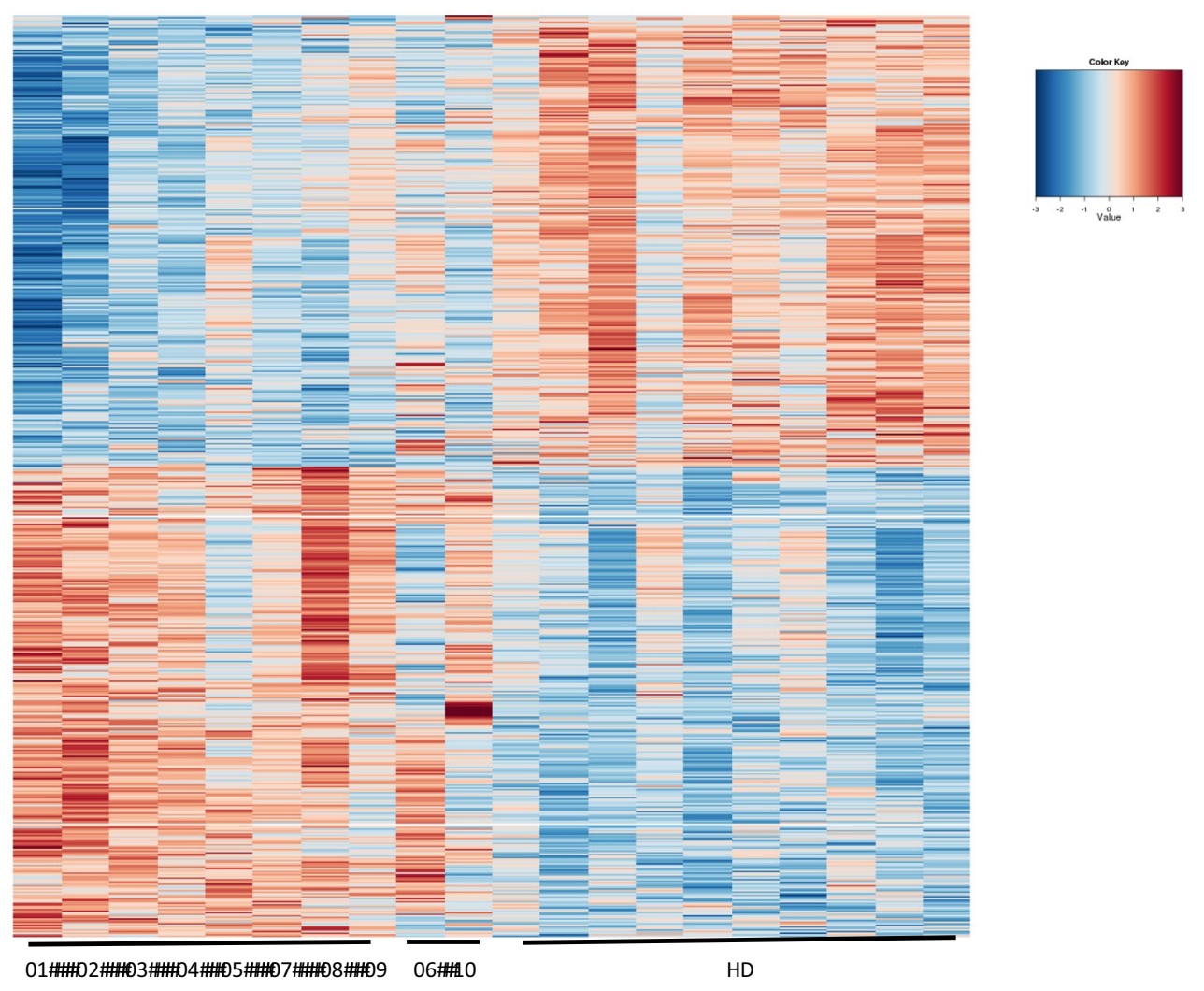


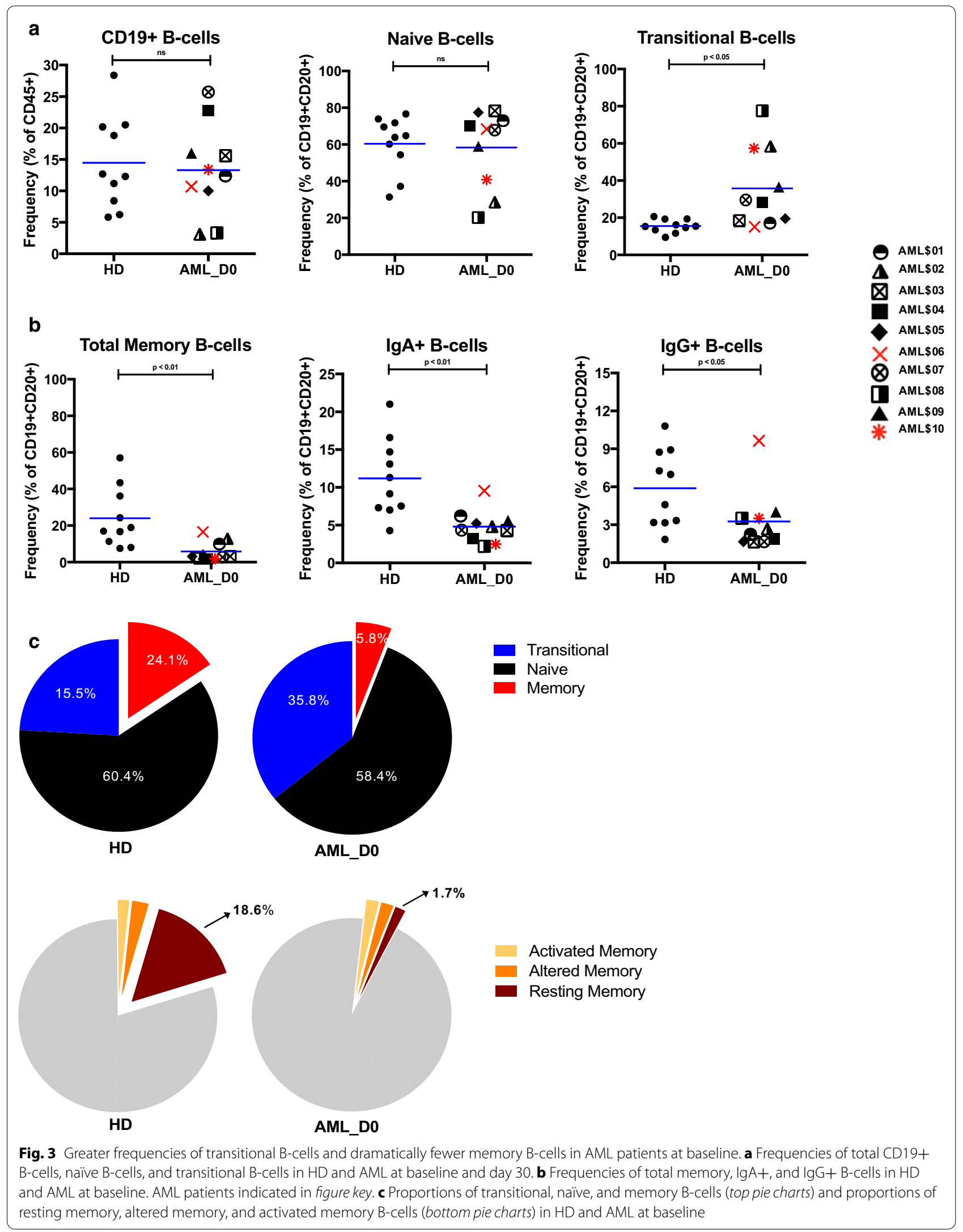


(resting memory, altered or tissue-like memory, and activated memory) reveals that resting memory B-cell (ID132) frequencies in AML are significantly reduced, being less than $10 \%$ of frequency seen in HD (mean frequency \pm SEM: $1.71 \% \pm 0.45$ vs. $18.5 \% \pm 4.29$ ) (Fig. 3c, bottom). Furthermore, the variance of frequencies of resting memory cells significantly differed between AML and HD ( $\mathrm{p}<0.05)$. We also noted reduced frequencies of CD86+ naïve (ID110) and memory B-cell populations (ID98) (Additional file 3: Figure S3) and several activated memory B-cell subsets with significantly different variances between AML and HD (summarized in Additional file 2: Table S4). We saw no significant differences between the means in any of these populations when comparing patients at baseline and 30 days post-vaccination (Additional file 3: Figure S4).

\section{Magnitude of B-cell subset abnormalities decline} with increasing time elapsed since chemotherapy As the time since the cessation of chemotherapy (or, the time since start of complete remission) varied across the 10 AML patients, we ranked these patients based on time elapsed since treatment and examined general trends of the B-cell populations over time, keeping in mind the heterogeneity of our cohort. Frequencies of transitional B-cells were lower in those patients sampled further from completion of chemotherapy (Fig. 4a). The three patients least removed from chemotherapy also had the lowest frequencies of naïve B-cells, suggesting rapid B-cell development in the 6 months after the cessation of treatment. Naïve B-cell frequencies were unchanged in patients 15 weeks or more post chemotherapy (Fig. 4b), while memory B-cells increased with time (Fig. 4c). Interestingly, frequencies of resting memory B-cells were well below the average \pm SEM in HD for all AML patients (Fig. 4d). While mature IgA+ B-cell frequencies also increased with time elapsed since chemotherapy (Fig. 4e), mature IgG+ B-cell frequency remained consistent across all patients in the first year after chemotherapy, and these patients had fewer frequencies of IgG+ B-cells than the average \pm SEM in HD (Fig. 4f). These trends indicate a return to healthy frequencies of transitional
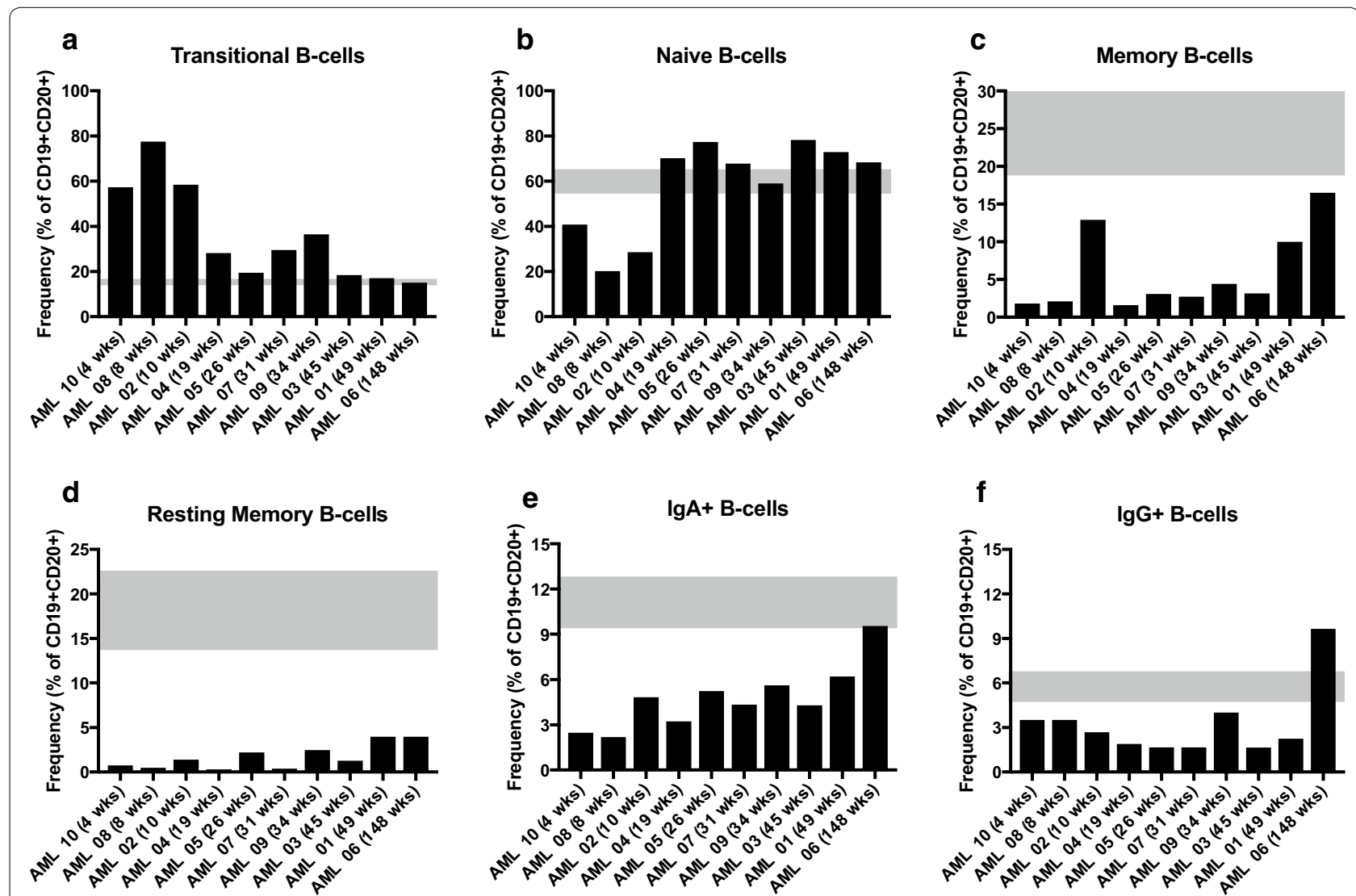

Fig. 4 Recovery of B-cell populations in AML patients is correlated with increasing time since chemotherapy. AML patients at baseline are ranked by time since end of chemotherapy and B-cell frequencies plotted. a Transitional B-cells. b Naïve B-cells. c Total memory B-cells. d Resting memory B-cells. e lgA+B-cells. f lgG+ B-cells. Gray boxes highlight mean values \pm SEM of the HDs 
and naïve B-cells roughly 6 months after the end of chemotherapy but a more uneven recovery of memory and effector B-cell subsets over time.

\section{B-cell repertoire is diverse, but antigen-inexperienced, in AML patients after chemotherapy}

To determine whether the B-cells from AML patients had molecular evidence of selection and mutation, we sequenced the B-cell receptor (BCR) complementaritydetermining region 3 (CDR3) region of the immunoglobulin heavy (IGH) chain. There were no differences in the ratios of productive to non-productive rearrangements ( $86 \%: 14 \%$ vs. $84 \%: 16 \%)$ or in overall clonality (0.029 vs. 0.030) in AML compared to HD (Additional file 3: Figure S5). We next looked at IGH CDR3 length, as this characteristic is important in determining BCR diversity. CDR3 length is approximately normally distributed in HD and in AML, with a few exceptions. Patients sampled at early time points post-chemotherapy have greater variability in CDR3 length and exhibited a shorter CDR3 loop distribution. The remaining patients who were 19 weeks or greater after completion of chemotherapy have CDR3 length distribution that closely aligns with that seen in HD (Additional file 3: Figure S6).

We considered the frequencies of distinct clonotypes with evidence of somatic hypermutation (SHM) in their IGH variable (IGHV) regions. We observed increased frequency of SHM in AML patients associated with time since last chemotherapy (Fig. 5a), and the percentage of distinct rearranged IGHV regions with evidence of SHM also roughly mirror the trends seen in the number of total memory B-cells (Fig. 4c). We delved deeper into the number of point mutations occurring in distinct clonotypes of B-cells of AML patients, and again noted that while AML patients least removed from chemotherapy had few to no CDR3 loops with more than one mutation, patients further into their remission demonstrated a greater number of point mutations in a greater number of clonotypes, indicative of somatic hypermutation (SHM) (Fig. 5b). Together, these data suggest that B-cell lymphopoiesis and affinity maturation begins after the cessation of chemotherapy, and the humoral immune system in these AML patients gradually increased the output of antigen-experienced memory B-cells in the first year of remission.




AML patients after consolidation chemotherapy demonstrated generally recovered $\mathrm{T}$-cell frequencies and function

AML patients in this study demonstrated no significant differences when compared to HD in frequencies (mean frequency \pm SEM) of total CD3+ (ID1) $(64.4 \% \pm 2.8$ vs. $57.5 \% \pm 2.8), \mathrm{CD} 4+$ (ID2) $(52.8 \% \pm 5.4$ vs. $56.1 \% \pm 3.8)$, or CD8+ (ID4) $(40.7 \% \pm 5.3$ vs. $35.5 \% \pm 3.3)$ T-cell populations (Fig. 6a). In addition, we observed no discernable changes or trends in any of these T-cell populations in AML patients between baseline and post-vaccination time points, nor when the patients were ranked by time elapsed since end of consolidation chemotherapy (Additional file 3: Figures S7, S8). The mean ratio of CD4+ to CD8+ T-cells for all 10 AML patients was 1.9-1, similar to the ratio seen in HD (1.8-1). Discrimination of CD4+ T-helper, and CD8+ T-cytotoxic into their naïve and memory subpopulations revealed no significant differences between the average frequencies seen in AML and $\mathrm{HD}$, although the AML patients did demonstrate greater heterogeneity (Additional file 3: Figures S9, S10). Of note, we observed highly varied distributions of several a

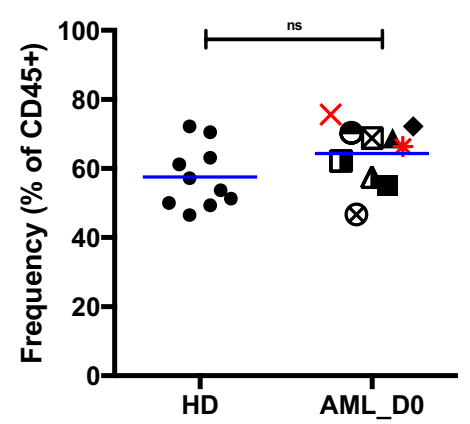

b

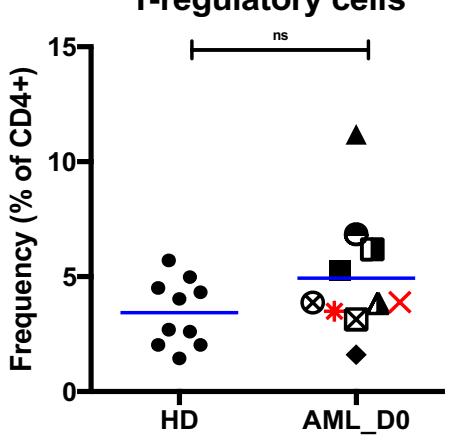

CD4+ T-cells

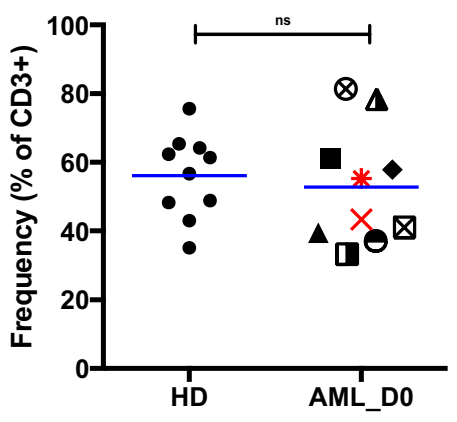

Naive T-reg cells

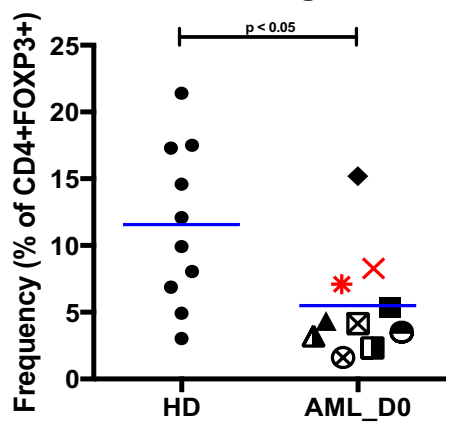

CD8+ T-cells

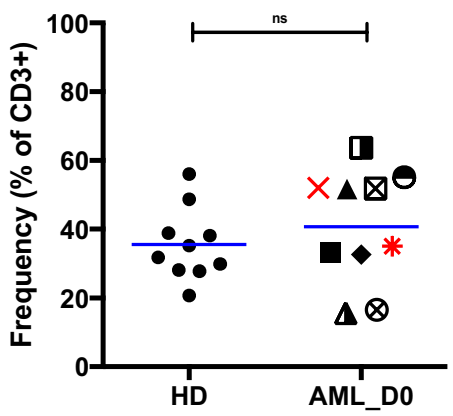

Memory T-reg cells

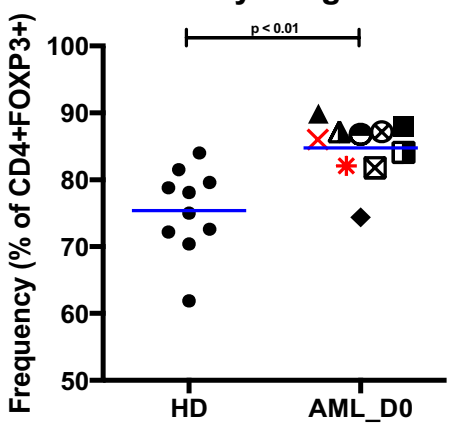

C

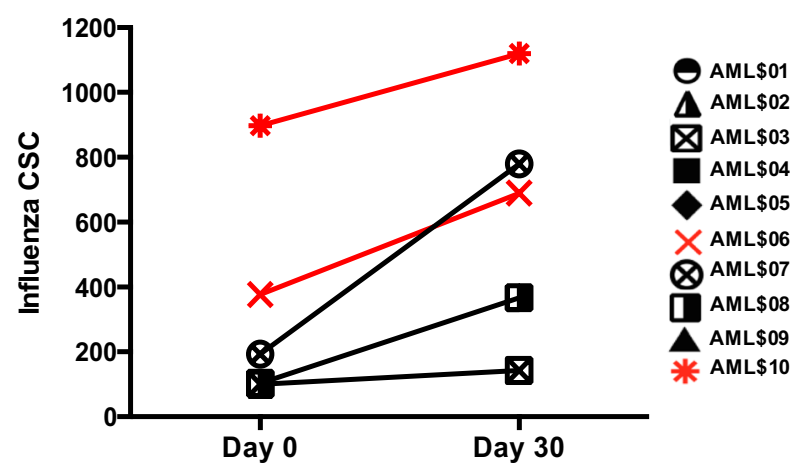

Fig. 6 Recovered T-cell frequencies and function at baseline in AML patients. a Frequencies of total CD3+, CD4+ T-helper, and CD8+ T-cytotoxic cells in HD and AML baseline. b Frequencies of total CD4+ FOXP3+ T-regs, naïve T-regs and of memory T-regs in HD and AML baseline. Mean frequencies indicated by blue lines. c Influenza-specific cytokine secretion on day 30 versus baseline in 5 evaluable AML patients, including two responders (red) and three non-responders (black). AML patients indicated in figure key 
cytotoxic CD8+ T-cell population frequencies in AML compared to HD (summarized in Additional file 2: Table S4).

We observed differences in many cell populations between AML and HD that include FOXP3+ T-regulatory cells (T-regs) (summarized in Additional file 2: Table S4). Frequencies of total T-regs (ID59) were similar between AML and HD (mean frequency \pm SEM: $4.9 \% \pm 0.8$ vs. $3.4 \% \pm 0.5$ ) (Fig. $6 \mathrm{~b}$, left panel). However, AML patients did have significantly reduced frequencies of naïve T-regs (ID129) $(5.5 \% \pm 1.3$ vs. $11.6 \% \pm 1.9$, $\mathrm{p}<0.05)$ and higher frequencies of memory T-regs (ID130) $(84.8 \% \pm 1.4$ vs. $75.4 \% \pm 2.0, \mathrm{p}<0.01)$ (Fig. $6 \mathrm{~b}$, middle and right panels) compared to HD.

We saw much variation but no discernable pattern in the over- or under-expressed frequencies of monocyte and dendritic cell populations between AML and HD (Fig. 2a; Additional file 2: Table S4). However, we did observe that the frequencies of conventional or myeloid dendritic cells (mDCs) (ID73) were lower in AML than in HD, while the frequencies of plasmacytoid dendritic cells (pDCs) (ID76) were higher (Additional file 3: Figure S11).

In addition to deep phenotypic assessment, we also directly assessed the functional capacity of T-cells in AML using IFN $\gamma$ T-cell ELISPOT assays in 5 patients who had available baseline and post-vaccination samples (including the 2 AML-R and 3 AML-NR). Surprisingly, all 5 patients saw increases in influenza-specific cytokine secretion after influenza vaccination (increases of 1.24to 4.0 -fold on day 30 over day 0) (Fig. 6c). Together, our data suggests that despite deficiencies in humoral immunity and apparent reduction of $\mathrm{mDC}$ frequencies, the T-cell compartment in AML patients has recovered as soon as 1 month post-consolidation and can generate a functional cellular immune response.

\section{Cell surface immune checkpoint expression is similar between AML patients in remission after chemotherapy and healthy donors}

Finally, as our protocol was designed to study a cohort of AML patients who may be considered as candidates for maintenance immunotherapies to prevent relapse, we sought to assess expression of cell surface immune checkpoint markers, namely, PD-1, TIM3, CTLA4, and $4-1 B B$ in AML at baseline and in healthy donors on various $\mathrm{CD} 8+\mathrm{T}$-cell populations. We found no differences in overall immune checkpoint marker expression, including double positive expression of these markers, between AML at baseline and HD on any CD8+ T-cell population. Of particular interest is PD-1 expression, and in AML and HD, respectively, we saw similarly varied frequencies (mean frequency \pm SEM) of PD-1+ total
CD8+ T-cells ( $16.6 \% \pm 4.6$ vs. $13.0 \% \pm 2.7), \mathrm{CD} 8+$ central memory ( $14.3 \% 4.0$ vs. $15.1 \%$ vs. 2.7$)$, CD8+ effector memory $(27.9 \% \pm 6.2$ vs. $28.9 \% \pm 5.8)$, and CD8+ terminal effector $(19.9 \% \pm 4.7$ vs. $13.1 \% \pm 3.1)(p>0.05$ for all) (Fig. 7a). Considering the AML patients based on time elapsed since treatment revealed that PD-1+ T-cells appeared to decrease in frequency with increasing time since chemotherapy (Fig. 7b-d).

\section{Discussion}

While immune system reconstitution after chemotherapy is important from an infectious disease standpoint, understanding the kinetics and biology behind this phenomenon is also important in the context of anticancer immunotherapy to prevent relapse and maintain remission in patients. Our goal with this study was to perform a deep assessment of the state of the adaptive immune system in AML patients in a first clinical remission after consolidation chemotherapy, not only to further the body of evidence for the timing of vaccinations against infectious disease in these patients, but also to characterize patients who may be considered candidates for post-chemotherapy immunotherapy. To do this, we sought to explore patterns of immune reconstitution following consolidation chemotherapy in AML patients in remission and to look for indications of a functional response, or lack thereof, to vaccination. Our approach, when compared to previous studies, was unique in that we established a comprehensive picture of the state of the adaptive "immunome" by simultaneously examining the genetic, phenotypic, and functional consequences of chemotherapy in AML patients. Importantly, we show a dramatic impact in the B-cell compartment, which appears slower to recover than the T-cell compartment after AML chemotherapy. We further show that the inability of AML patients to produce protective antibody titers in response to influenza vaccination is likely due to multiple B-cell abnormalities in this cohort of patients, including increased frequencies of transitional B-cells, a lack of affinity-matured, class-switched B-cells, and an antigen-inexperienced B-cell repertoire. Of particular interest are the generally recovered T-cell frequencies and the cytokine-secretion functionality of CD8+ $\mathrm{T}$-cells in response to influenza vaccination, suggesting that the two arms of the adaptive immune system are not equal in how they are affected by, or recover from, AML chemotherapy.

A recent study of post-vaccination responses to seasonal and pandemic influenza vaccination identified several immune subsets that were predictive of a robust and specific antibody response [17]. Among these predictive subsets, several CD38+ B-cell populations (ID91, ID96, ID103, ID108) were very highly correlated with a strong 




b

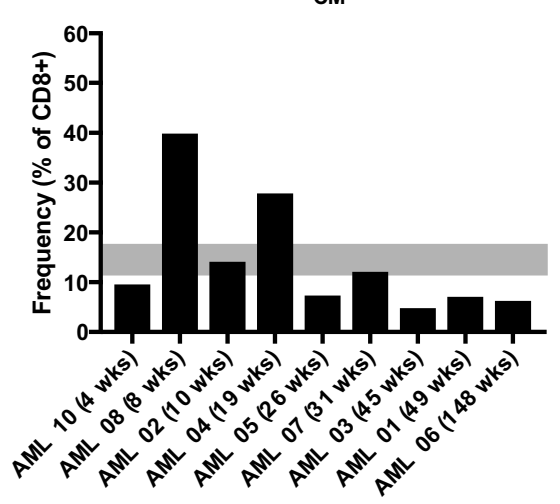

C

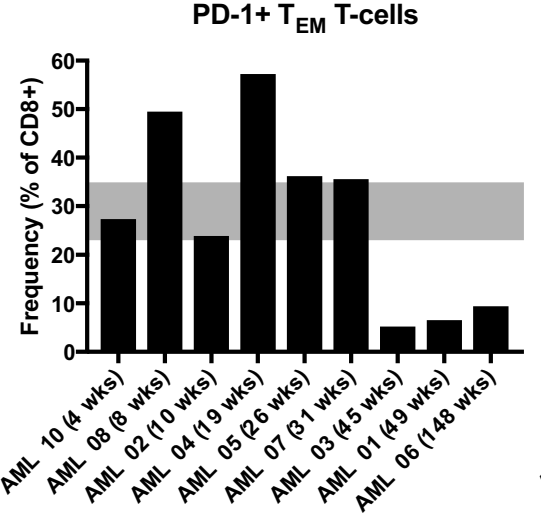

d

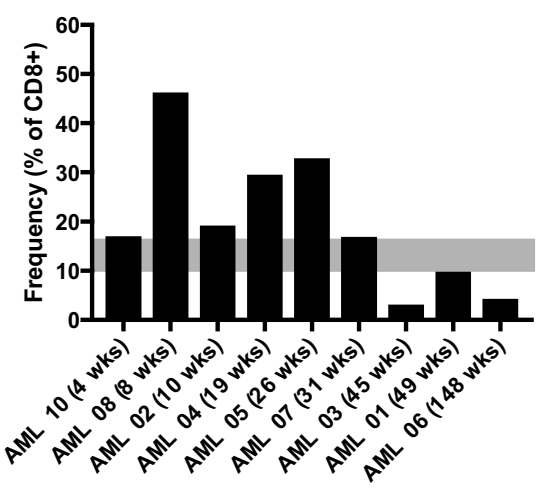

Fig. 7 PD-1 expression on CD8+ T-cell subsets is similar between HD and AML at baseline. a Frequencies of PD-1+ total CD8+, naïve (TN), central memory (TCM), effector memory (TEM), and terminal effector (TEMA $)$ CD8+ T-cell populations. AML patients and HD indicated in figure key. b-d AML patients at baseline are ranked by time since end of chemotherapy and frequencies of indicated PD-1+T-cell populations plotted. Gray boxes highlight mean values \pm SEM of the HDs

antibody response to influenza vaccination. However, in our data set, we saw no differences in the frequencies of these CD38+ populations in AML patients compared to $\mathrm{HD}$, nor did we see any difference in their frequencies between the AML-NR and AML-R. The lack of a relationship between these populations and response to vaccination in our AML cohort and HD indicate that applying a signature to predict post-vaccination responses is difficult in patients who receive chemotherapy, likely due to fluctuating immune parameters as immune reconstitution occurs. T-cell responses may be better surrogates of response to vaccination, especially in elderly individuals, but again, it has not been determined if this holds true in AML patients after they have received chemotherapy [26].

Previous studies in solid tumors and in lymphoid hematological malignancies have reported a delayed B-cell recovery and function during and in the year following allo-HSCT, cytotoxic chemotherapy, or B-cell depleting agents [27-29]. In a small cohort of pediatric AML patients either still receiving or having just completed 
treatment, it was shown that chemotherapy appeared especially deleterious for naïve and memory B-cells [28]. However, it remained unknown whether these effects would be the same in adult AML patients specifically in a complete remission post-consolidation. Furthermore, while both cellular and humoral immune reconstitution has been characterized in the allo-HSCT setting [29-35], it remained unclear what immune deficiencies could be ascribed to the consolidation chemotherapy patients received to achieve a $\mathrm{CR}$ before they receive conditioning therapy before transplant.

In our study, the total frequencies of CD19+ B-cells were similar between AML patients before vaccination and HD. The increased frequency of circulating immature, transitional B-cells suggest higher output by the bone marrow of these cells to repopulate the humoral immune niche [29], and the near absence of memory B-cell subsets and class-switched B-cells suggests either an inherently increased susceptibility of both memory and effector B-cells to chemotherapy, an insufficient amount of time to fully recover these cell populations, or both. CD27+ memory B-cells, and especially resting memory B-cells, are responsible for generating antibody responses, and circulating influenza-specific memory B-cells can last for decades [36-39]. The consistent finding of a reduction of memory B-cells in all the AML patients implicates this loss in the poor responses observed to influenza vaccination and suggests that humoral immunity reconstitution is a very long process.

The large number of differentially up-regulated genes in the 8 AML patients who did not respond to influenza vaccination compared to HD points towards a very active cell machinery across many cell pathways in various immune cell subsets. Furthermore, gene set enrichment analyses with very stringent criteria produced a very large number of up-regulated gene sets, underscoring the magnitude of perturbation in the intracellular state of PBMCs from the AML patients. The over-expression of genes related to cell death, metabolism, and genes more generally related to the immune response are strongly suggestive that the immune system is subject to lymphopoiesis and reconstitution in AML patients. Of particular interest is the upregulation of galectin-9 (LGALS9), which is the ligand for TIM-3 [40]. LGALS9 can exert pleiotropic effects through TIM-3, and it has been shown to promote leukemic stem cell renewal, suggesting that this gene that appears to be up-regulated by the process of immune reconstitution after chemotherapy may also have a role in AML relapse [41, 42].

Since the loss of memory B-cells is a central finding of our work, we sought to further interrogate the status of these cells through B-cell receptor IGH chain repertoire sequencing. The sequencing data shows that AML patients after chemotherapy have the same success rate at forming productive sequences as HD and can eventually form BCRs against specific antigen. Interestingly, the percentage of rearranged IGHV regions with point mutations indicative of somatic hypermutation in each patient tracked with the total frequency of memory B-cells, which were highest in frequency in patients furthest from last chemotherapy. Although we did not specifically look at B-cells involved in germinal center reactions as we did not sample peripheral lymphoid tissue in this study [43], our findings show that the humoral immune system in AML patients after chemotherapy is still capable of generating B-cell memory.

Frequencies of various T-cell subpopulations were grossly normal in our cohort of AML patients. The relatively recovered and intact $\mathrm{T}$-cell immune system in these patients and the ability of CD8+ T-cells to secrete IFN $\gamma$ in an antigenspecific manner suggest that $\mathrm{T}$-cell mediated immunotherapies are perhaps better suited at preventing and treating relapsed and refractory AML. We noted increased PD-1 expression on effector and memory T-cells shortly after the completion of chemotherapy in some of our AML patients. We believe there may be a window for immunotherapeutic intervention using immune checkpoint blockade to target residual disease in patients in remission. We do not fully address if the seemingly intact T-cell compartment in the blood of our patients were thymically derived or if surviving cells underwent peripheral expansion after chemotherapy. Recovery of CD4+ T-cells is thought to be generally thymus-independent, and one piece of corroborating evidence in our cohort lies in the higher frequency of memory T-regs and the reduced frequency of naïve T-regs in the AML patients, suggesting a proliferation of memory T-regs in the periphery as has been reported in the month immediately following chemotherapy $[15,44]$. However, the inverse has been reported in other hematological malignancies $[45,46]$, which has been ascribed to a reduced susceptibility of naïve T-regs to oxidative stress [47]. While T-regs are important in the maintenance of B-cell homeostasis and tolerance [48] in mice models receiving influenza vaccination, CD4+ FOXP3+ T-regs had no effect on B-cell responses [49]. However, this same study reported increased frequencies of T-regs in response to influenza vaccination, but we did not observe any appreciable increase in the frequencies of naïve or memory T-regs in our AML cohort after vaccination [49]. Thus, our observations on the relative frequencies of T-reg subsets in AML patients after chemotherapy differ somewhat with what has been reported in the literature, and more work into the underlying mechanisms of T-reg reconstitution and responses in the period immediately following chemotherapy and its effects on the success of nontransplant immunotherapeutic strategies is warranted.

A limitation of our work is the exclusion of CD161 and ICOS in our T-cell phenotyping panels, as recent 
reports identified populations of CD4+ CD161+ T-cells and ICOS+ CD38+ T-follicular helper cells that clonally expanded and persisted years after influenza vaccination in AML patients receiving chemotherapy and in healthy individuals receiving successive influenza vaccinations, respectively [50,51]. Additionally, the flow cytometric panels used in this study did not include natural killer (NK) cells. As a previous report identified recovered NK cell frequencies in an AML patient in remission [52], future planned studies will examine the frequencies of NK cells in AML patients in remission, as this cell population is an active area of exploration as an anti-leukemic immunotherapy [53]. Additionally, we will compare T-cells in the marrow compartment with those sampled from the blood to examine whether there are phenotypic and functional differences between cells from these two related yet distinct sites of disease. Furthermore, one consideration we have not addressed is the possibility of AML minimal residual disease (MRD) under the threshold of detection by morphologic examination contributing to local immunosuppression in the tumor microenvironment and its effect on lymphocyte recovery. It has been shown that AML blasts can induce IL-10 and IL-17 production by Th17 cells [54], and IL-10 can have differential effects on B-cells depending on their differentiation and activation status [55]. The effect of AML MRD on B-cell recovery in particular remains to be determined, and studying Th17 cells as well in the context of AML remission is also warranted.

While our study was small and our cohort of AML patients was diverse in terms of disease etiology, clinical outcomes, and duration into their first remission, we strikingly found similar patterns of immune dysfunction across all the patients. Notably, when we ranked the patients based on time elapsed since chemotherapy and used cell frequency trends as surrogates for immune reconstitution over time, the degree of dysfunction was less in those patients that were further away from chemotherapy. Our better understanding of the changes in adaptive immune cell subsets after chemotherapy in AML patients will be useful in designing immunotherapies that can work with existing immune capacity to eliminate residual disease and maintain a remission in patients with AML.

\section{Conclusions}

In conclusion, we have shown that impaired responses to influenza vaccination in our AML patients is associated with altered B-cell composition and impaired B-cell immunity. Successful responses to vaccinations require both functional cellular and humoral adaptive immunity, and the uncoupled B-cell and T-cell responses we have described have implications for the success of certain immunotherapies. These findings may have implications for the use of immunotherapy in AML.

\section{Additional files}

Additional file 1. Supplemental methods.

Additional file 2. Supplemental tables.

Additional file 3. Supplemental figures.

\begin{abstract}
Abbreviations
AML: acute myeloid leukemia; CR: complete remission; CR1: first complete remission; Allo-HSCT: allogeneic hematopoietic stem cell transplantation; HD: healthy donor; PBMCs: peripheral blood mononuclear cells; CSCs: cytokinesecreting T-cells; ASCs: antibody-secreting B-cells; APL: acute promyelocytic leukemia; DEG: differentially expressed gene; SEM: standard error of the mean; BCR: B-cell receptor; IGH: immunoglobulin heavy; IGHV: immunoglobulin heavy chain variable; CDR3: complementarity-determining region 3; SHM: somatic hypermutation; T-regs: T-regulatory cells.
\end{abstract}

\section{Authors' contributions}

MG performed experiments, analyzed data, wrote the manuscript, and made the figures. GP enrolled and cared for patients on the protocol and provided comments on the manuscript. AB, SM, LK, BS, JC, RS, HZ, HG, JM, and LK performed experiments and provided comments on the manuscript. FC and YK analyzed data and provided comments on the manuscript. KN collected and collated all the patient samples and provided comments on the manuscript. BDS and IMB supervised the clinical protocol and sample acquisition, contributed to study design, and edited the manuscript. CSH designed and supervised the research, wrote the clinical protocol, established the collaborations, and edited the manuscript. All authors read and approved the final manuscript.

\section{Author details \\ ${ }^{1}$ Myeloid Malignancies Section, Hematology Branch, National Heart, Lung and Blood Institute, National Institutes of Health, 10 Center Drive Room 10CRC 5-5216, Bethesda, MD 20814-1476, USA. ${ }^{2}$ Johns Hopkins University, Baltimore, MD, USA. ${ }^{3}$ Center for Human Immunology, Autoimmunity and Inflamma- tion, National Institutes of Health, Bethesda, MD, USA. ${ }^{4}$ National Institute of Allergy and Infectious Diseases, National Institutes of Health, Bethesda, MD, USA. ${ }^{5}$ Division of Viral Products, Center for Biologics Evaluation and Research, Food and Drug Administration, Silver Spring, MD, USA. ${ }^{6}$ Office of Biostatis- tics Research, National Heart, Lung, and Blood Institute, National Institutes of Health, Bethesda, MD, USA.}

\section{Acknowledgements}

The authors thank Jackie Greer for her coordination of research on the clinical trial, Jingrong Tang and Matthew Mule for their technical assistance, Kimberly Dunham for the T-cell ELISPOT data, Ervin Griffin, Andrea Casildo, and Lakshmi Rudraraju for sample processing, and the NHLBI Flow Cytometry Core, especially Philip McCoy and Pradeep Dagur, for assistance in setting up immune checkpoint flow cytometry panels. The authors also thank Laura Dillon and Katherine Lindblad for reviewing the manuscript.

The manuscript is dedicated to Dr. Robert ("Bob") Nussenblatt, Associate Director of the NIH Center for Human Immunology. Were it not for his untimely passing in 2016 he would have been a co-author of this work.

Meghali Goswami is a pre-doctoral candidate in the Molecular Medicine Program of the Institute for Biomedical Sciences at The George Washington University. This work is from a dissertation to be presented to the above program in partial fulfillment of the requirements for the Ph.D. degree.

\section{Competing interests}

CSH reports that his laboratory receives research funding from SELLAS Life Sciences AG and Merck. The other authors declare no competing financial interests. 


\section{Availability of data and materials}

Primary microarray expression data have been deposited in the Gene Expression Omnibus (GEO) under accession GSE97485. All other datasets generated and analyzed during the current study are available from the corresponding author upon reasonable request.

\section{Ethics approval and consent to participate}

Protocol J1293 "Cellular and molecular characterization of the immune response before and after immunization with the seasonal influenza vaccine in adult patients who have completed treatment for acute myeloid leukemia" was approved by Sidney Kimmel Comprehensive Cancer Center at Johns Hopkins IRB on 8/31/2012 (study ID NA_00077854_AM00031358). All patients signed written consent.

\section{Funding}

This research was supported by the Intramural Research Program of the National Heart, Lung, and Blood Institute of the National Institutes of Health.

\section{Publisher's Note}

Springer Nature remains neutral with regard to jurisdictional claims in published maps and institutional affiliations.

Received: 18 May 2017 Accepted: 21 June 2017

Published online: 10 July 2017

\section{References}

1. Welch JS, Ley TJ, Link DC, Miller CA, Larson DE, Koboldt DC, et al. The origin and evolution of mutations in acute myeloid leukemia. Cell. 2012;150(2):264-78

2. Ramos NR, Mo CC, Karp JE, Hourigan CS. Current approaches in the treatment of relapsed and refractory acute myeloid leukemia. J Clin Med. 2015;4(4):665-95.

3. Koreth J, Schlenk R, Kopecky KJ, Honda S, Sierra J, Djulbegovic BJ, et al. Allogeneic stem cell transplantation for acute myeloid leukemia in first complete remission: systematic review and meta-analysis of prospective clinical trials. JAMA. 2009;301(22):2349-61.

4. Hourigan CS, Levitsky HI. Evaluation of current cancer immunotherapy: hemato-oncology. Cancer J. 2011;17(5):309-24

5. Hourigan CS, McCarthy P, de Lima M. Back to the future! The evolving role of maintenance therapy after hematopoietic stem cell transplantation. Biol Blood Marrow Transpl. 2014;20(2):154-63.

6. Lichtenegger FS, Krupka C, Kohnke T, Subklewe M. Immunotherapy for acute myeloid leukemia. Semin Hematol. 2015;52(3):207-14.

7. Rezvani K, Yong AS, Tawab A, Jafarpour B, Eniafe R, Mielke S, et al. Ex vivo characterization of polyclonal memory CD8+ T-cell responses to PRAMEspecific peptides in patients with acute lymphoblastic leukemia and acute and chronic myeloid leukemia. Blood. 2009;113(10):2245-55.

8. Busse A, Letsch A, Scheibenbogen C, Nonnenmacher A, Ochsenreither S, Thiel $E$, et al. Mutation or loss of Wilms' tumor gene 1 (WT1) are not major reasons for immune escape in patients with AML receiving WT1 peptide vaccination. J Transl Med. 2010;8:5.

9. Keilholz U, Letsch A, Busse A, Asemissen AM, Bauer S, Blau IW, et al. A clinical and immunologic phase 2 trial of Wilms tumor gene product 1 (WT1) peptide vaccination in patients with AML and MDS. Blood. 2009;113(26):6541-8.

10. Uttenthal B, Martinez-Davila I, Ivey A, Craddock C, Chen F, Virchis A, et al. Wilms'tumour 1 (WT1) peptide vaccination in patients with acute myeloid leukaemia induces short-lived WT1-specific immune responses. Br J Haematol. 2014;164(3):366-75.

11. Maslak PG, Dao T, Krug LM, Chanel S, Korontsvit T, Zakhaleva V, et al. Vaccination with synthetic analog peptides derived from WT1 oncoprotein induces T-cell responses in patients with complete remission from acute myeloid leukemia. Blood. 2010;116(2):171-9.

12. Rezvani K, Yong AS, Mielke S, Jafarpour B, Savani BN, Le RQ, et al. Repeated PR1 and WT1 peptide vaccination in Montanide-adjuvant fails to induce sustained high-avidity, epitope-specific CD8+ T cells in myeloid malignancies. Haematologica. 2011;96(3):432-40.
13. Kuball J, de Boer K, Wagner E, Wattad M, Antunes E, Weeratna RD, et al. Pitfalls of vaccinations with WT1-, Proteinase3- and MUC1-derived peptides in combination with MontanidelSA51 and CpG7909. Cancer Immunol Immunother. 2011;60(2):161-71.

14. Rosenblatt J, Stone RM, Uhl L, Neuberg D, Joyce R, Levine JD, et al. Individualized vaccination of $A M L$ patients in remission is associated with induction of antileukemia immunity and prolonged remissions. Sci Transl Med. 2016;8(368):368ra171.

15. Kanakry CG, Hess AD, Gocke CD, Thoburn C, Kos F, Meyer C, et al. Early lymphocyte recovery after intensive timed sequential chemotherapy for acute myelogenous leukemia: peripheral oligoclonal expansion of regulatory T cells. Blood. 2011;117(2):608-17.

16. Nakaya HI, Hagan T, Duraisingham SS, Lee EK, Kwissa M, Rouphael N, et al. Systems analysis of immunity to influenza vaccination across multiple years and in diverse populations reveals shared molecular signatures. Immunity. 2015;43(6):1186-98.

17. Tsang JS, Schwartzberg PL, Kotliarov Y, Biancotto A, Xie Z, Germain RN, et al. Global analyses of human immune variation reveal baseline predictors of postvaccination responses. Cell. 2014;157(2):499-513.

18. Kunisaki KM, Janoff EN. Influenza in immunosuppressed populations: a review of infection frequency, morbidity, mortality, and vaccine responses. Lancet Infect Dis. 2009;9(8):493-504.

19. Beck CR, McKenzie BC, Hashim AB, Harris RC, Zanuzdana A, Agboado G, et al. Influenza vaccination for immunocompromised patients: systematic review and meta-analysis from a public health policy perspective. PLoS ONE. 2011;6(12):e29249.

20. Rowe T, Abernathy RA, Hu-Primmer J, Thompson WW, Lu X, Lim W, et al. Detection of antibody to avian influenza A (H5N1) virus in human serum by using a combination of serologic assays. J Clin Microbiol. 1999;37(4):937-43.

21. Biancotto A, Dagur PK, Fuchs JC, Wiestner A, Bagwell CB, McCoy JP Jr. Phenotypic complexity of $T$ regulatory subsets in patients with B-chronic lymphocytic leukemia. Modern Pathol. 2012;25(2):246-59.

22. Biancotto A, Fuchs JC, Williams A, Dagur PK, McCoy JP Jr. High dimensional flow cytometry for comprehensive leukocyte immunophenotyping (CLIP) in translational research. J Immunol Methods. 2011;363(2):245-61.

23. Perfetto SP, Ambrozak D, Nguyen R, Chattopadhyay P, Roederer M. Quality assurance for polychromatic flow cytometry. Nat Protoc. 2006;1 (3):1522-30.

24. Carlson CS, Emerson RO, Sherwood AM, Desmarais C, Chung MW, Parsons $\mathrm{JM}$, et al. Using synthetic templates to design an unbiased multiplex PCR assay. Nat Commun. 2013;4:2680.

25. Robins HS, Campregher PV, Srivastava SK, Wacher A, Turtle CJ, Kahsai O, et al. Comprehensive assessment of T-cell receptor beta-chain diversity in alphabeta T cells. Blood. 2009;114(19):4099-107.

26. McElhaney JE, Xie D, Hager WD, Barry MB, Wang Y, Kleppinger A, et al. T cell responses are better correlates of vaccine protection in the elderly. J Immunol. 2006;176(10):6333-9.

27. Bedognetti D, Zoppoli G, Massucco C, Zanardi E, Zupo S, Bruzzone A, et al. Impaired response to influenza vaccine associated with persistent memory B cell depletion in non-Hodgkin's lymphoma patients treated with rituximab-containing regimens. J Immunol. 2011;186(10):6044-55.

28. Reilly A, Kersun LS, Luning Prak E, Boyer J, McDonald K, Jawad AF, et al. Immunologic consequences of chemotherapy for acute myeloid leukemia. J Pediatr Hematol Oncol. 2013;35(1):46-53.

29. Mensen A, Johrens K, Anagnostopoulos I, Demski S, Oey M, Stroux A, et al. Bone marrow T-cell infiltration during acute GVHD is associated with delayed B-cell recovery and function after HSCT. Blood. 2014;124(6):963-72

30. Avanzini MA, Locatelli F, Dos Santos C, Maccario R, Lenta E, Oliveri M, et al. $B$ lymphocyte reconstitution after hematopoietic stem cell transplantation: functional immaturity and slow recovery of memory CD27+ B cells. Exp Hematol. 2005;33(4):480-6.

31. Xhaard A, Moins-Teisserenc H, Busson M, Robin M, Ribaud P, Dhedin N, et al. Reconstitution of regulatory T-cell subsets after allogeneic hematopoietic SCT. Bone Marrow Transplant. 2014;49(8):1089-92.

32. Servais $S$, Lengline $E$, Porcher $R$, Carmagnat $M$, Peffault de Latour $R$, Robin $M$, et al. Long-term immune reconstitution and infection burden after mismatched hematopoietic stem cell transplantation. Biol Blood Marrow Transpl. 2014;20(4):507-17.

33. Bosch M, Khan FM, Storek J. Immune reconstitution after hematopoietic cell transplantation. Curr Opin Hematol. 2012;19(4):324-35. 
34. Douek DC, Vescio RA, Betts MR, Brenchley JM, Hill BJ, Zhang L, et al. Assessment of thymic output in adults after haematopoietic stemcell transplantation and prediction of T-cell reconstitution. Lancet. 2000;355(9218):1875-81.

35. D'Orsogna LJ, Wright MP, Krueger RG, McKinnon EJ, Buffery SI, Witt CS, et al. Allogeneic hematopoietic stem cell transplantation recipients have defects of both switched and igm memory B cells. Biol Blood Marrow Transpl. 2009;15(7):795-803.

36. Moir S, Ho J, Malaspina A, Wang W, DiPoto AC, O'Shea MA, et al. Evidence for HIV-associated B cell exhaustion in a dysfunctional memory B cell compartment in HIV-infected viremic individuals. J Exp Med. 2008;205(8):1797-805

37. Tangye SG, Avery DT, Deenick EK, Hodgkin PD. Intrinsic differences in the proliferation of naive and memory human B cells as a mechanism for enhanced secondary immune responses. J Immunol. 2003;170(2):686-94.

38. Good KL, Avery DT, Tangye SG. Resting human memory B cells are intrinsically programmed for enhanced survival and responsiveness to diverse stimuli compared to naive B cells. J Immunol. 2009;182(2):890-901.

39. Yu X, Tsibane T, McGraw PA, House FS, Keefer CJ, Hicar MD, et al. Neutralizing antibodies derived from the B cells of 1918 influenza pandemic survivors. Nature. 2008;455(7212):532-6.

40. Zhu C, Anderson AC, Schubart A, Xiong H, Imitola J, Khoury SJ, et al. The Tim-3 ligand galectin-9 negatively regulates $T$ helper type 1 immunity. Nat Immunol. 2005;6(12):1245-52.

41. Kikushige Y, Akashi K. TIM-3 as a therapeutic target for malignant stem cells in acute myelogenous leukemia. Ann NY Acad Sci. 2012;1266:118-23.

42. Kikushige Y, Miyamoto T, Yuda J, Jabbarzadeh-Tabrizi S, Shima T, Takayanagi S, et al. A TIM-3/Gal-9 autocrine stimulatory loop drives self-renewal of human myeloid leukemia stem cells and leukemic progression. Cell Stem Cell. 2015;17(3):341-52.

43. Kolar GR, Mehta D, Pelayo R, Capra JD. A novel human B cell subpopulation representing the initial germinal center population to express AID. Blood. 2007;109(6):2545-52.

44. Mackall CL, Fleisher TA, Brown MR, Andrich MP, Chen CC, Feuerstein IM, et al. Age, thymopoiesis, and CD4+ T-lymphocyte regeneration after intensive chemotherapy. N Engl J Med. 1995;332(3):143-9.
45. Beyer M, Schultze JL. Regulatory T cells in cancer. Blood. 2006;108(3):804-11.

46. Kordasti SY, Ingram W, Hayden J, Darling D, Barber L, Afzali B, et al. CD4+ CD25high Foxp3 + regulatory $T$ cells in myelodysplastic syndrome (MDS). Blood. 2007;110(3):847-50.

47. Mougiakakos D, Johansson CC, Kiessling R. Naturally occurring regulatory T cells show reduced sensitivity toward oxidative stress-induced cell death. Blood. 2009;113(15):3542-5.

48. Kinnunen T, Chamberlain N, Morbach H, Choi J, Kim S, Craft J, et al. Accumulation of peripheral autoreactive $B$ cells in the absence of functional human regulatory T cells. Blood. 2013;121(9):1595-603.

49. Surls J, Nazarov-Stoica C, Kehl M, Casares S, Brumeanu TD. Differential effect of CD4+ Foxp3+ T-regulatory cells on the B and Thelper cell responses to influenza virus vaccination. Vaccine. 2010;28(45):7319-30.

50. Alsuliman A, Muftuoglu M, Khoder A, Ahn YO, Basar R, Verneris MR, et al. A subset of virus-specific CD161+ T cells selectively express the multidrug transporter MDR1 and are resistant to chemotherapy in AML. Blood. 2016:129:740

51. Herati RS, Muselman A, Vella L, Bengsch B, Parkhouse K, Del Alcazar D, et al. Successive annual influenza vaccination induces a recurrent oligoclonotypic memory response in circulating T follicular helper cells. Sci Immunol. 2017;2(8). doi:10.1126/sciimmunol.aag2152.

52. Braciak TA, Wildenhain S, Roskopf CC, Schubert IA, Fey GH, Jacob U, et al. NK cells from an AML patient have recovered in remission and reached comparable cytolytic activity to that of a healthy monozygotic twin mediated by the single-chain triplebody SPM-2. J Transl Med. 2013;11:289.

53. Muntasell A, Lopez-Botet M. Natural killer cell-based immunotherapy in acute myeloid leukemia: lessons for the future. Clin Cancer Res. 2016;22(8):1831-3.

54. Musuraca G, De Matteis S, Napolitano R, Papayannidis C, Guadagnuolo $V$, Fabbri F, et al. IL-17/IL-10 double-producing T cells: new link between infections, immunosuppression and acute myeloid leukemia. J Transl Med. 2015;13:229.

55. Itoh K, Hirohata S. The role of IL-10 in human B cell activation, proliferation, and differentiation. J Immunol. 1995;154(9):4341-50.

\section{Submit your next manuscript to BioMed Central and we will help you at every step:}

- We accept pre-submission inquiries

- Our selector tool helps you to find the most relevant journal

- We provide round the clock customer support

- Convenient online submission

- Thorough peer review

- Inclusion in PubMed and all major indexing services

- Maximum visibility for your research

Submit your manuscript at www.biomedcentral.com/submit
() Biomed Central 